\title{
ANESTESIA TOTAL INTRAVENOSA (TIVA) ASSOCIADA AO BLOQUEIO DO PLEXO BRAQUIAL EM UM OVINO SUBMETIDO À OSTEOSSÍNTESE DE RÁDIO E ULNA - RELATO DE CASO
}

\author{
SANCHES, Guilherme Lessa ${ }^{1}$ \\ CALABREZ, Larissa Pim ${ }^{1}$ \\ STOCCO, Anieli Vidal ${ }^{1}$ \\ BOLZAN, Thays Carvalho de Amorim ${ }^{1}$ \\ DI FILIPPO, Paula Alessandra ${ }^{1}$ \\ ANTUNES, Fernanda ${ }^{1}$
}

\section{Recebido em: 2020.04.25 Aprovado em: $2020.11 .03 \quad$ ISSUE DOI: $10.3738 / 21751463.3786$}

\begin{abstract}
RESUMO: A anestesia total intravenosa (TIVA) é uma técnica que veņ sendo utilizada na prática anestésica veterinária, principalmente em pequenos animais. Seu emprego se popularizou na última década, devido aos novos estudos utilizando fármacos mais adequados, com menores efeitos cumulativos e menor tempo de recuperação anestésica. Objetivou-se avaliar a infusão de propofol juntamente com o bloqueio do plexo braquial em um ovino submetido a osteossíntese de rádio e ulna. A pré-medicação anestésica foi realizada com cetamina $10 \%$ na dose de 2 $\mathrm{mg} / \mathrm{Kg}$, a indução com propofol $1 \%$ na dose de $5 \mathrm{mg} / \mathrm{Kg}$ e a manutenção anestésica com propofol na dose de $0,25 \mathrm{mg} / \mathrm{Kg} / \mathrm{min}$. O bloqueio do plexo braquial foi realizado com a associação de lidocaína 2\% e bupivacaína 0,5\%, ambas na dose de $0,12 \mathrm{ml} / \mathrm{Kg}$, com um volume final de $20 \mathrm{ml}$. Foram avaliados durante todo o procedimento: frequência cardíaca $(\mathrm{FC})$, frequência respiratória $(f)$, saturação periférica de oxigênio na hemoglobina $\left(\mathrm{SpO}_{2}\right)$ pressão arterial sistólica (PAS), pressão arterial diastólica (PAD), pressão arterial média (PAM), temperatura retal (T), reflexo palpebral, reflexo corneal e a profundidade anestésica. Os parâmetros foram anotados antes da medicação pré-anestésica (T0), imediatamente após a indução anestésica (T10), e depois, a cada 10 minutos, até o final da infusão (T90). Todos os parâmetros cardiorrespiratórios avaliados limitaram-se ao fisiológico para a espécie. Conclui-se que o protocolo de TIVA utilizado no ovino promoveu anestesia para realização da osteossíntese, juntamente ao bloqueio do plexo braquial, que foi essencial para a analgesia e para manutenção anestésica adequada.
\end{abstract}

Palavras-chave: Infusão contínua. Pequenos ruminantes. Propofol.

\section{TOTAL INTRAVENOUS ANESTHESIA (TIVA) ASSOCIATED WITH BRACHIAL PLEXUS BLOCKING IN AN OVINE SUBJECT TO RADIO AND ULNA OSTEOSYNTHESIS - CASE REPORT}

\begin{abstract}
SUMMARY: Total intravenous anesthesia (TIVA) is a technique that has been used in veterinary anesthetic practice, mainly in small animals. Its use has become popular in the last decade, due to new studies using more suitable drugs, with less cumulative effects and shorter anesthetic recovery time. The objective of this study was to evaluate the propofol infusion together with the brachial plexus block in an ovine submitted to radius and ulna osteosynthesis. Anesthetic premedication was performed with $10 \%$ ketamine at a dose of $2 \mathrm{mg} / \mathrm{kg}$, induction with $1 \%$ propofol at a dose of $5 \mathrm{mg} / \mathrm{kg}$ and anesthetic maintenance with propofol at a dose of $0.25 \mathrm{mg} / \mathrm{kg} / \mathrm{min}$. The brachial plexus block was performed with the combination of $2 \%$ lidocaine and $0.5 \%$ bupivacaine, both at a dose of $0.12 \mathrm{ml} / \mathrm{kg}$, with a final volume of $20 \mathrm{ml}$. During the whole procedure, heart rate (HR), respiratory rate $(f)$, peripheral oxygen saturation in hemoglobin (SpO2), systolic blood pressure (SBP), diastolic blood pressure (DBP), mean arterial pressure (MAP), temperature were evaluated during the entire procedure. rectal (T), eyelid reflex, corneal reflex and anesthetic depth. The parameters were recorded before pre-anesthetic medication (T0), immediately after anesthetic induction (T10), and then, every 10 minutes, until the end of the infusion (T90). All cardiorespiratory parameters evaluated were limited to the physiological for the species. It is concluded that the TIVA protocol used in the sheep promoted anesthesia to perform osteosynthesis, together with brachial plexus block, which was essential for analgesia and for adequate anesthetic maintenance.
\end{abstract}

Keywords: Continuous infusion. Small ruminants. Propofol.

\footnotetext{
${ }^{1}$ Universidade Estadual do Norte Fluminense Darcy Ribeiro, Campos dos Goytacazes, RJ, Brasil - Laboratório de Clínica e Cirurgia Animal (LCCA)
}

Nucleus Animalium, v.12, n.2, nov. 2020 


\section{INTRODUÇÃO}

Procedimentos cirúrgicos realizados em ovinos vêm crescendo, principalmente em animais com alto valor zootécnico (LIZARRAGA; CHAMBERS, 2012). Os pequenos ruminantes se destacam como modelos experimentais em muitos procedimentos cirúrgicos por apresentarem porte adequado, maior facilidade de manuseio, serem de fácil aquisição, além de apresentarem respostas hemodinâmicas farmacológicas semelhantes ao ser humano (ADAMS; MCKINLEY, 2009).

Em relação às técnicas para manutenção anestésica, temos a anestesia inalatória, anestesia parcial intravenosa (PIVA) e a anestesia total intravenosa (TIVA), esta última produz plano anestésico adequado, reduz a poluição do ambiente cirúrgico e não necessita de aparelhagem específica quando comparada à anestesia inalatória. O propofol é indicado por ser um fármaco de curta duração, permitindo uma recuperação mais suave com mínimos efeitos excitatórios e rápido metabolismo extra-hepático (CORREIA et al.,1996)

Nos ovinos, uma das primeiras avaliações clínicas utilizando o propofol foi realizada para indução anestésica e manutenção da anestesia em infusão contínua (WATERMAN, 1988; MATHER, 1989). Associada a anestesia geral, podem ser empregadas técnicas de anestesia locorregional, como o bloqueio do plexo braquial. Seu emprego permite a realização de procedimentos cirúrgicos distais à articulação do cotovelo (FERNANDES et al., 2019).

Considerando o número ainda pequeno de trabalhos com relatos sobre a aplicação prática do bloqueio do plexo braquial e da infusão contínua de propofol em pequenos ruminantes, o objetivo do presente trabalho foi avaliar os efeitos da associação dessas duas técnicas em um ovino submetido a osteossíntese de rádio e ulna.

\section{RELATO DE CASO}

Um ovino, da raça Dorper, macho, com $80 \mathrm{~kg}, 5$ anos, foi atendido com o histórico de trauma no membro torácico direito, provocado por outro ovino da mesma raça. O paciente apresentava edema da região afetada, normorexia, normodpsia, normoúria, normofagia. Na avaliação pré-anestésica foi observado leucocitose neutrofílica sem desvio à esquerda e no exame bioquímico, não havia nenhuma alteração.

Após 24 horas de jejum sólido e 12 horas de jejum líquido, o paciente foi preparado para o procedimento cirúrgico. Foi puncionada a veia jugular externa direita para introdução de cateter 16G no qual foi administrada a medicação pré-anestésica (MPA), composta de cetamina $2 \mathrm{mg} / \mathrm{kg}$. 
Logo após o início da sedação, o animal foi posicionado em decúbito lateral esquerdo e foi realizada tricotomia de todo membro torácico direito.

Em seguida, iniciou-se a administração de ringer com lactato na taxa de $10 \mathrm{ml} / \mathrm{kg} / \mathrm{h}$. O bloqueio do plexo braquial foi realizado pela técnica descrita por Santos, Trein e Luna (2019), sem o auxílio de eletroestimulador ou ultrassonografia. Foram identificadas as estruturas anatômicas e foi introduzida a agulha para anestesia locorregional, na borda cranial e caudo ventral ao colo da escapula na região infraescapular em direção à região onde o plexo braquial se forma. A agulha foi introduzida $15 \mathrm{~cm}$ e imediatamente foi depositado todo o volume de anestésico local em leque. Para realização do bloqueio do plexo braquial foi utilizado lidocaína $2 \%$ na dose de $0,12 \mathrm{ml} / \mathrm{Kg}$ e bupivacaína $0,5 \%$ na dose de $0,12 \mathrm{ml} / \mathrm{kg}$ (Figura 1 ).

Figura 1 - Realização do bloqueio do plexo braquial. Animal em decúbito lateral esquerdo, a cabeça (A) no inferior da imagem. Mão esquerda segurando a articulação do ombro do membro torácico direito, enquanto a mão direita introduz a agulha na região no plexo, para deposição do anestésico local.

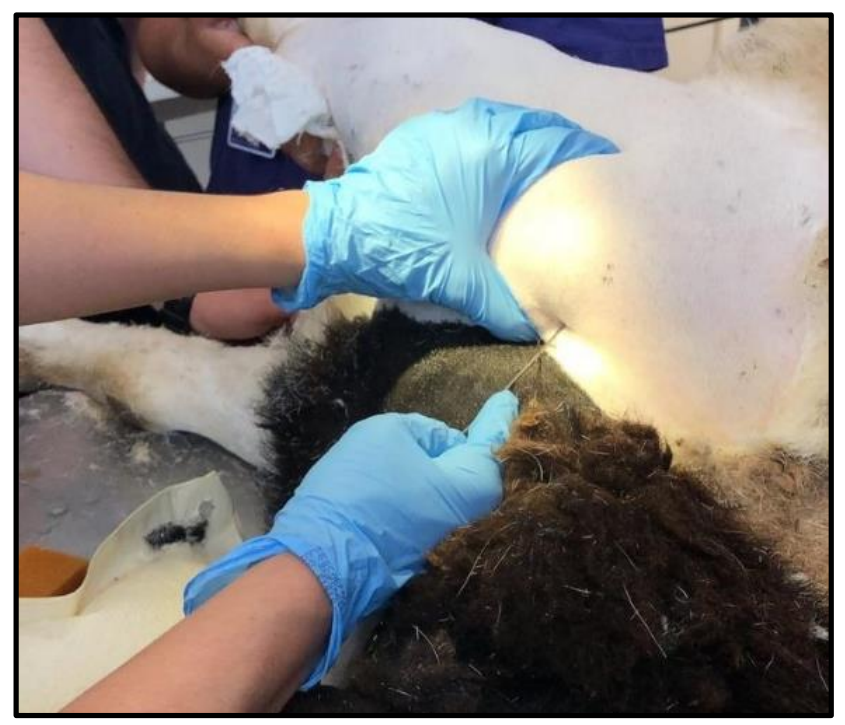

Fonte: arquivo pessoal.

Com isso, foi então realizada a indução anestésica com propofol na dose de $5 \mathrm{mg} / \mathrm{kg}$, até que fosse permitido a intubação endotraqueal. Com o auxílio de um laringoscópio de lâmina longa, foi realizado o abaixamento da língua e visualização da epiglote e da entrada da laringe, sendo utilizada uma sonda endotraqueal do tipo Murphy, tamanho 10, para intubação. O paciente foi posicionado na mesa cirúrgica em decúbito dorsal com o membro torácico direito elevado e foi conectado ao circuito circular, recebendo oxigênio $100 \%$ com fluxo de $20 \mathrm{ml} / \mathrm{kg} / \mathrm{min}$, em respiração espontânea. A cabeça foi posicionada de forma que ficasse menos elevada que o restante do corpo, para facilitar a saída de secreções respiratórias/gástricas da cavidade oral e das vias áreas superiores. 
A partir daí, iniciou-se a infusão contínua de propofol na dose de $0,25 \mathrm{mg} / \mathrm{kg} / \mathrm{min}$, na bomba de seringa Terumo TE-331. Foi conectado ao paciente o equipamento de monitoração anestésica - monitor InMonitor-InPulse. Foram avaliados frequência cardíaca (FC), saturação periférica de oxigênio na hemoglobina $\left(\mathrm{SpO}_{2}\right)$, pressão arterial sistólica (PAS), pressão arterial diastólica (PAD), pressão arterial média (PAM) e eletrocardiograma (ECG). Também foram monitorados a frequência respiratória $f$ (através da observação dos movimentos do gradil costal), a temperatura retal em graus Celsius, o reflexo palpebral, o reflexo corneal e o plano anestésico. Os parâmetros foram anotados antes da MPA (T0), imediatamente após a indução com propofol (T10) e, depois, a cada 10 min, até a interrupção da infusão do propofol (T90). O procedimento cirúrgico iniciou-se no momento T20 e terminou no momento T90. A infusão foi iniciada imediatamente após a indução e teve duração total de 70 minutos (Tabela 1).

Tabela 1 - Parâmetros avaliados em um ovino pré-medicado com $2 \mathrm{mg} / \mathrm{Kg}$ de cetamina, recebendo infusão contínua de propofol na dose de $0,25 \mathrm{mg} / \mathrm{kg} / \mathrm{min}$, associado ao bloqueio do plexo braquial e submetido a osteossíntese de rádio e ulna.

\begin{tabular}{|c|c|c|c|c|c|c|c|c|c|c|}
\hline & T0 & T10 & T20 & T30 & T40 & T50 & T60 & T70 & T80 & T90 \\
\hline FC & 96 & 80 & 87 & 90 & 85 & 80 & 86 & 82 & 90 & 80 \\
\hline $\mathbf{F}$ & 68 & 45 & 43 & 42 & 40 & 38 & 30 & 33 & 30 & 30 \\
\hline $\mathrm{SpO2}$ & - & 95 & 97 & 95 & 94 & 96 & 95 & 97 & 93 & 94 \\
\hline PAS & - & 120 & 117 & 111 & 106 & 98 & 100 & 110 & 90 & 98 \\
\hline PAD & - & 72 & 80 & 78 & 87 & 71 & 79 & 80 & 66 & 70 \\
\hline PAM & - & 88 & 92 & 89 & 93 & 80 & 86 & 90 & 74 & 79 \\
\hline $\mathbf{T}^{\circ} \mathbf{C}$ & 38,3 & 38 & 37,6 & 37,5 & 37,3 & 37,3 & 37 & 37 & 36,8 & 36,9 \\
\hline $\begin{array}{l}\text { Reflexo } \\
\text { palpebral }\end{array}$ & $\mathrm{P}$ & PL & PL & PL & PL & PL & PL & PL & PL & PL \\
\hline $\begin{array}{l}\text { Reflexo } \\
\text { corneal }\end{array}$ & $\mathrm{P}$ & $\mathrm{P}$ & $\mathrm{P}$ & $\mathrm{P}$ & $\mathrm{P}$ & $\mathrm{P}$ & $\mathrm{P}$ & $\mathrm{P}$ & $\mathrm{P}$ & $\mathrm{P}$ \\
\hline $\begin{array}{l}\text { Plano } \\
\text { anestésico }\end{array}$ & - & A & A & A & A & A & A & A & A & A \\
\hline
\end{tabular}

Legenda: Frequência cardíaca (FC) e respiratória $(f)$, saturação periférica de oxigênio na hemoglobina (SpO2), pressão arterial sistólica (PAS), pressão arterial diastólica (PAD), pressão arterial média (PAM), temperatura corpórea $(\mathrm{T})$, reflexo palpebral e reflexo corneal $(\mathrm{A}=$ Ausente/P=Presente/PL=Presente e lento) $\mathrm{e}$ plano anestésico $(\mathrm{A}=$ Adequado/S=Superficial/P=Profundo). Fonte: Elaboração própria.

O procedimento foi finalizado sem nenhuma intercorrência e em seguida, foi levado para a baia de internação, onde foi acompanhado até a recuperação. O tempo desde a última dose de propofol até a permanência em decúbito esterno abdominal e início da ruminação foi de 35 min. $\mathrm{Na}$ baia de internação recebeu butorfanol 0,1mg/kg BID, dipirona $25 \mathrm{mg} / \mathrm{kg}$ e flumexine meglumine $1,1 \mathrm{mg} / \mathrm{Kg}$, durante 5 dias. 


\section{DISCUSSÃO}

Quando o procedimento cirúrgico não permite que o animal permaneça em estação, é recomendado que o paciente fique em declive num ângulo de 15 a $20^{\circ}$, com a cabeça mais baixa, ou então seja utilizado algum objeto por baixo do pescoço, que impeça o retorno do alimento e mantenha a cabeça reclinada para baixo, possibilitando a drenagem das secreções (MASSONE, 2017). Todos os cuidados relacionados ao jejum e ao posicionamento do paciente na mesa, evitaram a regurgitação transoperatória, promovendo maior segurança no ato anestésico.

A MPA foi suficiente para causar contenção química no paciente e permitir a realização do bloqueio do plexo braquial. A dose de $2 \mathrm{mg} / \mathrm{Kg}$ de cetamina também foi utilizada por Góss et al. (2016) em um ovino submetido à remoção de um higroma na região do carpo, promovendo imobilidade por 30 minutos. A MPA raramente é necessária e muitas vezes não desejável em pequenos ruminantes, pois aumenta a incidência de regurgitação, além de prolongar a recuperação. Porém, na grande parte dos casos, as vantagens de seu uso superam as desvantagens (GALATOS, 2011).

O bloqueio do plexo braquial promove anestesia a partir da região distal do úmero, onde são insensibilizados os ramos ventrais dos três últimos nervos cervicais e os dois primeiros nervos torácicos. Na literatura, são encontrados relatos do bloqueio do plexo braquial em caprinos e ovinos, guiados por eletroestimulação e com o auxílio da ultrassonografia. (KAPRAL et al. 2008; GOEBEL, et al. 2010). Porém, nesse caso, o bloqueio foi realizado a partir de marcos anatômicos, conforme descrito por Santos, Trein e Luna (2019), os quais afirmam que a técnica pode ser empregada em bezerros e pequenos ruminantes, introduzindo 12 a $14 \mathrm{~cm}$ de uma agulha com $150 \mathrm{~mm}$ na altura do acrômio no sentido caudoventral até tocar o terço médio da primeira costela, depositando $20 \mathrm{ml}$ de anestésico local em leque nessa região, conforme realizado no caso descrito.

Em ruminantes, a lidocaína a 1 ou $2 \%$ e a bupivacaína a 0,25 ou $0,5 \%$ são os anestésicos mais utilizados. A seleção dos anestésicos locais deve levar em consideração o tempo cirúrgicoalvo. A lidocaína apresenta período de latência curto e período de ação moderado; já a bupivacaína, período de latência maior, tendo uma ação mais prolongada, que pode alcançar até 360 minutos (SANTOS; TREIN; LUNA, 2019). A escolha dos anestésicos locais utilizados no bloqueio do plexo braquial foi em razão de sua farmacologia, permitindo então que o procedimento fosse realizado sem estímulo álgico nos primeiros minutos pela ação mais instantânea da lidocaína e o conforto pós-operatório fosse instituído pela ação da bupivacaína.

O sucesso do bloqueio deve-se ao fato dos parâmetros vitais terem permanecido dentro dos intervalos normais para a espécie durante todo procedimento, mesmo o animal se mantendo 
em um plano superficial de anestesia geral, além do fato de não ter sido utilizado nenhum outro analgésico potente anterior ou durante o procedimento cirúrgico.

De acordo com Galatos (2011), a indução com a administração intravenosa de anestésicos injetáveis é preferível a indução com a administração intramuscular ou anestésicos inalatórios, pois reduz a incidência de refluxo e aspiração de conteúdo ruminal. Essa constatação vai ao encontro de Gozalo-Marcilla, Nóbrega Neto e Luna (2019), que afirmam ser possível realizar a indução anestésica com agentes injetáveis ou inalatórios, embora as vias intravenosa e intramuscular sejam as mais adequadas. Neste caso, a via intravenosa e o fármaco propofol foram escolhidos, mas o tiopental, a cetamina e éter gliceril guaiacol também poderiam ser empregados em determinadas situações.

Conforme Gozalo-Marcilla, Nóbrega Neto e Luna (2019), a intubação endotraqueal com balonete em todos os ruminantes é recomendada para proteger as vias áreas do conteúdo ruminal regurgitado e da saliva, além de possibilitar a administração de oxigênio e agentes inalatórios, quando for o caso. Em ovinos, pode ser realizada com laringoscópio de lâmina longa, com o animal em decúbito esterno abdominal e a cabeça e pescoço estendidos. A intubação do paciente foi realizada em decúbito dorsal com o auxílio de um laringoscópio adaptado, possibilitando visualizar a epiglote e a entrada da laringe, permitindo o fornecimento de oxigênio e evitando uma possível broncoaspiração, uma vez que, o paciente apresentou intensa sialorréia.

Em relação ao acompanhamento anestésico, a oximetria de pulso reflete a porcentagem de oxihemoglobina saturada por oxigênio, que proporciona alto grau de previsibilidade da hipoxemia e possibilita identificar a redução na saturação muito antes de iniciar a cianose (NUNES, 2010; CARREGARO; SILVA, 2019). Existe uma correlação direta da $\mathrm{SpO}_{2}$ com a $\mathrm{PaO}_{2}$ (pressão parcial de oxigênio no sangue arterial). Assim, os valores de $\mathrm{SpO}_{2}$ acima de $95 \%$ refletem valores de $\mathrm{PaO}_{2}$ acima de $80 \mathrm{mmHg}$ (CARREGARO; SILVA, 2019). No caso descrito, a saturação se manteve acima de $95 \%$ na maior parte do transoperatório, pressupondo, desta maneira, adequada oxigenação tecidual. Somente em um momento foi registrado o valor de $93 \%$, e em outros dois momentos $94 \%$ provavelmente por algum artefato de movimentação do sensor, luz ambiente, pela intensa salivação, ou então pela $\mathrm{PaO}_{2}$ abaixo de $80 \mathrm{mmHg}$.

Para Freitas e Carregaro (2019), a FC em ovinos varia de 80 a 115 bpm, a PAS de 120 a $160 \mathrm{mmHg}$, a PAD de 60 a $70 \mathrm{mmHg}$ e a PAM de 80 a $90 \mathrm{mmHg}$. A FC, a PAS e a PAD mantiveram-se dentro dos limites para espécie durante todos os momentos. Somente a PAM, nos momentos T80 e T90, teve ligeira redução, porém nada muito significativo em relação aos valores normais para a espécie (Figura 5 e 6). Lukarsewski (2011), em um relato utilizando TIVA com propofol e cetamina em um ovino, também observou a manutenção dos valores de FC e de 
pressão arterial dentro dos limites para a espécie. Em outro estudo realizado por De Lima et al. (2016), utilizando TIVA com propofol em ovinos pré-medicados com detomidina, foram observados valores de FC abaixo dos valores de referência, provavelmente devido ao uso da detomidina, que promove bradicardia.

Durante toda anestesia intravenosa, os ruminantes também necessitam da suplementação de oxigênio (RIEBOLD, 1996). O paciente relatado recebeu $100 \%$ de oxigênio através do tubo endotraqueal durante todo o procedimento. A $f$ em ruminantes varia de 20 a 40 mov/min (RIEBOLD, 2002), porém a frequência se manteve acima desses valores em T10, T20 e T30 (Figura 7). O mesmo foi observado por Correia et al. (1996) em ovinos sob infusão de propofol e cetamina, que mantiveram a $f$ variando entre 26 e $45 \mathrm{mov} / \mathrm{min}$. Situação diferente foi observada no relato de Lukarsewski (2011) cuja $f$ se manteve em 4 mov/min em ventilação controlada, porém sem prejuízos nas variáveis de hemogasometria.

A hipotermia é frequente em paciente submetidos a anestesia devido a depressão da função de termorregulação hipotalâmica, associada a perdas de calor pelas condições do ambiente e do procedimento cirúrgico. Essa condição reduz o metabolismo das células, promove bradicardia e retarda a recuperação anestésica. Por ser de difícil reversão, é melhor evitá-la (CARREGARO; SILVA, 2019). Como aponta Yasbek (2010), a temperatura em carneiros adultos oscila entre 38,5 e $39,8{ }^{\circ} \mathrm{C}$, com média de $39,3{ }^{\circ} \mathrm{C}$. Situações nas quais a temperatura fica abaixo de $36,0^{\circ} \mathrm{C}$, a hipotermia passa a ter importância clínica. A partir do momento T10, a temperatura do caso em estudo já estava abaixo de de $38,5^{\circ} \mathrm{C}$, sendo que em T80 foi registrado o menor valor de temperatura, porém em nenhum momento ficou inferior a zona crítica

O ECG é a única monitoração que identifica alterações de condução do impulso elétrico, ou seja, arritmias. As informações obtidas pelo ECG refletem apenas a função de atividade elétrica do coração e não são precisas sobre as variáveis hemodinâmicas (CARREGARO; SILVA, 2019). Durante todo transoperatório, o traçado eletrocardiográfico demonstrou ritmo sinusal, não ocorrendo nenhum episódio de arritmia.

O reflexo palpebral desaparece com o mínimo aprofundamento da anestesia em bovinos, ovinos e cabras. Quando o paciente está sob anestesia cirúrgica, a íris e a pupila centralizam-se entre as duas pálpebras e, ao mínimo aprofundamento anestésico, o globo ocular rotaciona ventralmente. Determinar a profundidade anestésica apenas com a rotação ventral do globo ocular não é suficiente para procedimentos mais invasivos, já o reflexo corneal deve estar sempre presente nessas espécies. Por outro lado, movimentos intencionais, em todas as espécies, indicam inadequada profundidade anestésica (RIEBOLD, 2002).

Dessa forma, o ovino em análise permaneceu com o reflexo palpebral medial lento, juntamente com o reflexo corneal, indicando plano anestésico superficial, porém, nesse caso, o 
episódio foi visto como adequado, devido a associação do bloqueio do plexo braquial. Em um relato realizado por Lukarsewski (2011) sobre um ovino mantido em infusão de propofol e cetamina, com bloqueio epidural, visando a abordagem da cavidade abdominal, também foi mantido um plano anestésico superficial, permanecendo o paciente com o reflexo palpebral presente. Em outro trabalho realizado por De Lima et al. (2016), o bloqueio epidural para cirurgia de tíbia em ovinos que receberam infusão contínua de propofol, também manteve os animais em plano cirúrgico superficial, com o reflexo palpebral medial presente.

$\mathrm{Na}$ situação estudada no presente trabalho, a manutenção da taxa de propofol em 0,25 $\mathrm{mg} / \mathrm{Kg} / \mathrm{min}$ propiciou plano anestésico superficial, porém suficiente para manter o animal inconsciente e imóvel. Presume-se que a realização do bloqueio locorregional do plexo braquial permitiu a manutenção da dose reduzida de infusão do propofol, mantendo o paciente em um plano mais superficial. Isso foi observado por De Lima et al. (2016), que realizaram o bloqueio peridural em ovinos para cirurgia de tíbia e verificaram que a mesma taxa de $0,25 \mathrm{mg} / \mathrm{Kg} / \mathrm{min}$ foi suficiente para a promover inconsciência e imobilidade.

\section{CONCLUSÃO}

A partir dos resultados obtidos, pode-se concluir que a infusão de propofol no ovino prémedicado com cetamina, manteve a estabilidade cardiovascular e promoveu anestesia geral para a realização da cirurgia de osteossíntese do rádio e ulna do paciente, sem nenhuma intercorrência. Entretanto, a realização do bloqueio do plexo braquial associado à anestesia total intravenosa foi essencial para adequada analgesia durante este procedimento cirúrgico.

\section{REFERÊNCIAS}

ADAMS, D.; MCKINLEY, M. The sheep. Anzccart Humane Science, v. 9, p.1-13, 2009.

BOUDREAU, A.E. et al. A comparison of 3 anesthetic protocols for 24 hours of mechanical ventilation in cats. Journal of Veterinary Emergency and Critical Care. v. 22, n. 2, p. 39-52, 2012.

CARREGARO, A.B; SILVA, A.N.E. Monitoração anestésica. In: LUNA, S.P.L; CARREGARO, A.B. Anestesia e Analgesia em equídeos, ruminantes e suínos.1.ed. São Paulo: Medvep, p.247280, 2019.

CORREIA, D. et al. Pharmacokinetics of propofol infusions, either alone or with ketamine, in sheep premedicated with acepromazine and papaveretum. Research In Veterinary Science., v.60, p.213-217, 1996. 
DE LIMA, M. P. A. et al. Propofol or midazolam infusion associated with subarachnoid anaesthesia in sheep submitted to bilateral tibial osteotomy. Brazilian Journal of Veterinary Medicine. v. 38, n. 3, p. 250-256, 2016.

FERNANDES, N.S. et al. Bloqueio de plexo braquial guiado por neuroestimulador para amputação em bezerro. Acta Scientiae Veterinariae. v. 47, p.398, 2019.

FREITAS, G.C; CARREGARO, A.B. Avaliação Pré-anestésica e Morbidade Anestesica. In: LUNA, S.P.L; CARREGARO, A.B. Anestesia e Analgesia em equídeos, ruminantes e suínos. São Paulo: Medvep, 2019. p.226-245.

GALATOS, A.D. Anesthesia and Analgesia in Sheep and Goats. Veterinary Clinics Food Animal, v. 27, p. 47-59, 2011.

GOEBEL, S. et al. Interscalene brachial plexus block for open-shoulder surgery: a randomized, double-blind, placebo-controlled trial between singleshot anesthesia and patient-controlled catheter system. Archives of Orthopaedic and Trauma Surgery. v.130. n. 4, p. 533-540, 2010.

GÓSS G.C. et al. Carpal hygroma in one sheep - Case report. Brazilian Journal of Veterinary Medicine. v. 38, n. 4, p. 445-448, 2016.

GOZALO-MARCILLA, M; NÓBREGA NETO, P.I; LUNA, S.P.L. Anestesia intravenosa e inalatória em ruminantes. In: LUNA, S.P.L; CARREGARO, A.B. Anestesia e Analgesia em equídeos, ruminantes e suínos. São Paulo: Medvep,, 2019. p. 512-531.

KAPRAL, S. et al. Ultrasonographic guidance improves the success rate of interscalene brachial plexus blockade. Regional Anesthesia and Pain Medicine. v. 33, n.3, p. 253-258, 2008.

LIZARRAGA, I.; CHAMBERS, J.P. Use of analgesic drugs for pain managment in sheep. New Zealand Veterinary Journal. v. 60, p. 87-94, 2012.

LUKARSEWSKI, R. Anestesia intravenosa total com propofol e cetamina em ovino prémedicado com cetamina, midazolam e tramadol - relato de caso. Monografia de residéncia medico veterinária. Universidade Federal de Santa Maria, Rio grande do Sul, 2011.

MASSONE, F. Anestesiologia Veterinária: farmacologia e técnicas: texto e atlas colorido. 6.ed. Rio de Janeiro: Guanabara Koogan, 2017. 467 p.

MATHER, L.E. et al. Propofol: assay and regional mass balance in the sheep. Xenobiotica. v.19, n.20, p. 133, 1989.

NUNES, N. Monitoração da Anestesia. In: NUNES, N.; FANTONI, D.T.; CORTOPASSI, S.R.G. Anestesia em Cães e Gatos. 2 ed. São Paulo: Ed. Roca, p.83-101, 2010.

RIEBOLD, T.W. Ruminant Anesthesia. In: RIEBOLD, T.W.; GREENE, S. Veterinary Anesthesia and Pain Management Secrets. 1 ed. Philadelphia: Hanley \& Belfus, Inc., p. 253262,2002

RIEBOLD, T.W. Ruminants. In THURMON, J.C., TRANQUILLI, W.J., BENSON,G.J Lumb \& Jones' Veterinary Anesthesia, 3 ed. Baltimore: Williams \& Wilkins, 1996. p. 610-624. 
SANTOS, P.S.P., TREIN., T.A., LUNA, S.P.L. Anestesia Locorregional em Ruminantes e Suínos. In: LUNA, S.P.L; CARREGARO, A.B. Anestesia e Analgesia em equídeos, ruminantes e suínos. São Paulo: Medvep, 2019. p.397-436

WATERMAN, A. Use of propofol in sheep. Vet. Rec. v. 122, n.11, p. 260, 1988.

YASBEK, K.V.B. Hipotermia. In:_YASBEK, K.V.B.; FANTONI, D.T.; CORTOPASSI, S.R.G. Anestesia em Cães e Gatos. 2 ed. São Paulo: Ed. Roca, 2010. p.605-610. 